\title{
Extracting Faint Signals Behind Bright Sources From CLASH Imaging Using Fourier Analysis
}

\author{
Yao Li ${ }^{1, a}$, Ding-Peng Li ${ }^{1, b}$, Feng-Jie Lei ${ }^{2, c}$ and Feng-Shan Liu ${ }^{1,2, d^{*}}$ \\ ${ }^{1}$ College of Physical Science and Technology, Shenyang Normal University, Shenyang 110034, \\ China \\ ${ }^{2}$ National Astronomical Observatories, Chinese Academy of Sciences, A20 Datun Road, Beijing \\ 100012, China

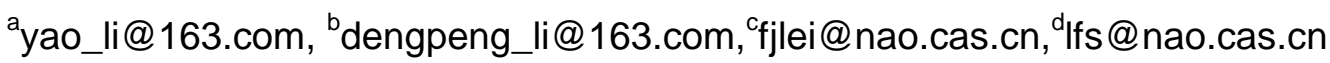

\begin{abstract}
Keywords: CLASH, Image processing, Intra-cluster light, Fourier expansion
Abstract. With the combination of deep Hubble Space Telescope Imaging and magnification due to gravitational lensing, the CLASH program offers an unprecedented opportunity to search for supernovae at high redshifts and detect some of the most distant galaxies as well. Unfortunately, the cluster field suffers from a high abundance of bright foreground sources and high background due to intra-cluster light. To overcome these difficulties, we have developed a method of modeling and subtracting bright foreground light in the clusters using Fourier analysis. In this paper, I will present preliminary results based our method, which significantly increases the effective depth of the images, and efficiently characterizes distant background signals in the clusters.
\end{abstract}

\section{Introduction}

The Cluster Lensing and Supernova survey with Hubble (CLASH) is one of three Hubble Multi-Cycle Treasury (MCT) programs selected in 2009, which observes massive galaxy clusters at intermediate redshifts. With the combination of deep Hubble Space Telescope Imaging and magnification due to cluster gravitational lensing, the CLASH program has four main science goals: (1) to measure the profiles and substructures of dark matter in galaxy clusters with unprecedented precision and resolution, (2) to discover and monitor distant supernovae in the cluster fields through a staggered program of multi-wavelength observations, (3) to characterize some of the most distant galaxies yet discovered, and (4) to study the internal structure and evolution of the galaxies in and behind these intermediate clusters [1].

Unfortunately, the region of clusters is a crowed field and foreground cluster members are usually much brighter than distant background sources. Moreover, a substantial fraction of stars in clusters are not gravitationally bound to any particular cluster galaxy. These stars constitute the so-called intra-cluster light (ICL). The ICL is distributed around the central dominated galaxy of the cluster and extends to several hundred $k p c$ away from the cluster center. This diffuse light contributes to a relatively high background, which suppresses the weak signals of sources in the early universe [2]. Therefore, in order to detect and characterize these faint background sources, an efficient method is needed to model and subtract the foreground light in the clusters.

In this paper, we report a new method of modeling and subtracting the foreground light in the clusters using Fourier analysis, which was developed by our group independently. The outline of the paper is as follows. In subsequent two sections, we describe the imaging data of Hubble Space Telescope in CLASH and our modeling, respectively. A conclusion is drawn in the last section.

\section{HST Imaging Data in CLASH}

The CLASH sample contains 25 clusters of galaxies with $\mathrm{z}=0.18-0.89$ and global X-ray temperatures greater than $5 \mathrm{keV}$. Twenty of these clusters were selected for their X-ray surface brightness symmetry indicating relaxed clusters, and the remaining five were specifically selected to 
Table 1 - The CLASH Cluster Sample and HST Observing Plan

\begin{tabular}{lcccccc}
\hline \hline \multicolumn{1}{c}{ Cluster } & $\alpha_{\mathrm{J} 2000}$ & $\delta_{\mathrm{J} 2000}$ & $\mathrm{z}_{\text {Clus }}$ & $\begin{array}{c}\text { HST } \\
\text { Cycle }\end{array}$ & Orbits & $\begin{array}{c}\text { Program } \\
\text { ID }\end{array}$ \\
\hline Abell 209 & $01: 31: 52.57$ & $-13: 36: 38.8$ & 0.206 & 19 & 20 & 12451 \\
Abell 383 & $02: 48: 03.36$ & $-03: 31: 44.7$ & 0.187 & 18 & 20 & 12065 \\
MACS0329.7-0211 & $03: 29: 41.68$ & $-02: 11: 47.7$ & 0.450 & 19 & 20 & 12452 \\
MACS0429.6-0253 & $04: 29: 36.10$ & $-02: 53: 08.0$ & 0.399 & 20 & 20 & 12788 \\
MACS0744.9+3927 & $07: 44: 52.80$ & $+39: 27: 24.4$ & 0.686 & 18 & 17 & 12067 \\
Abell 611 & $08: 00: 56.83$ & $+36: 03: 24.1$ & 0.288 & 19 & 18 & 12460 \\
MACS1115.9+0129 & $11: 15: 52.05$ & $+01: 29: 56.6$ & 0.352 & 19 & 20 & 12453 \\
Abell 1423 & $11: 57: 17.26$ & $+33: 36: 37.4$ & 0.213 & 20 & 20 & 12787 \\
MACS1206.2-0847 & $12: 06: 12.28$ & $-08: 48: 02.4$ & 0.440 & 18 & 20 & 12069 \\
CLJ1226.9+3332 & $12: 26: 58.37$ & $+33: 32: 47.4$ & 0.890 & 20 & 18 & 12791 \\
MACS1311.0-0310 & $13: 11: 01.67$ & $-03: 10: 39.5$ & 0.494 & 20 & 20 & 12789 \\
RXJ1347.5-1145 & $13: 47: 30.59$ & $-11: 45: 10.1$ & 0.451 & 18 & 15 & 12104 \\
MACS1423.8+2404 & $14: 23: 47.76$ & $+24: 04: 40.5$ & 0.545 & 20 & 17 & 12790 \\
RXJ1532.9+3021 & $15: 32: 53.78$ & $+30: 20: 58.7$ & 0.345 & 19 & 20 & 12454 \\
MACS1720.3+3536 & $17: 20: 16.95$ & $+35: 36: 23.6$ & 0.391 & 19 & 20 & 12455 \\
Abell 2261 & $17: 22: 27.25$ & $+32: 07: 58.6$ & 0.224 & 18 & 20 & 12066 \\
MACS1931.8-2635 & $19: 31: 49.66$ & $-26: 34: 34.0$ & 0.352 & 19 & 20 & 12456 \\
RXJ2129.7+0005 & $21: 29: 39.94$ & $+00: 05: 18.8$ & 0.234 & 19 & 20 & 12457 \\
MS2137-2353 & $21: 40: 15.18$ & $-23: 39: 40.7$ & 0.313 & 18 & 18 & 12102 \\
RXJ2248.7-4431 & $22: 48: 44.29$ & $-44: 31: 48.4$ & 0.348 & 19 & 20 & 12458 \\
MACS0416.1-2403 & $04: 16: 09.39$ & $-24: 04: 03.9$ & 0.42 & 19 & 20 & 12459 \\
MACS0647.8+7015 & $06: 47: 50.03$ & $+70: 14: 49.7$ & 0.584 & 18 & 18 & 12101 \\
MACS0717.5+3745 & $07: 17: 31.65$ & $+37: 45: 18.5$ & 0.548 & 18 & 17 & 12103 \\
MACS1149.6+2223 & $11: 49: 35.86$ & $+22: 23: 55.0$ & 0.544 & 18 & 18 & 12068 \\
MACS2129.4-0741 & $21: 29: 26.06$ & $-07: 41: 28.8$ & 0.570 & 18 & 18 & 12100 \\
\hline
\end{tabular}

Table 2-CLASH Observing Information

\begin{tabular}{lccccc}
\hline \hline Camera/Channel & Filter & Orbits & $\begin{array}{c}\text { ExposureTime } \\
{[\mathrm{sec}]}\end{array}$ & $\begin{array}{c}10 \sigma \text { Limit } \\
{[\mathrm{ABmag}]}\end{array}$ & $\begin{array}{c}5 \sigma \text { Limit } \\
{[\mathrm{AB} \mathrm{mag}]}\end{array}$ \\
\hline WFC3/UVIS & F225W & 1.5 & 3558 & 25.7 & 26.4 \\
WFC3/UVIS & F275W & 1.5 & 3653 & 25.7 & 26.5 \\
WFC3/UVIS & F336W & 1.0 & 2348 & 25.9 & 26.6 \\
WFC3/UVIS & F390W & 1.0 & 2350 & 26.5 & 27.2 \\
ACS/WFC & F435W & 1.0 & 1984 & 26.4 & 27.2 \\
ACS/WFC & F475W & 1.0 & 1994 & 26.8 & 27.6 \\
ACS/WFC & F606W & 1.0 & 1975 & 26.9 & 27.6 \\
ACS/WFC & F625W & 1.0 & 2008 & 26.4 & 27.2 \\
ACS/WFC & F775W & 1.0 & 2022 & 26.2 & 27.0 \\
ACS/WFC & F814W & 2.0 & 4103 & 27.0 & 27.7 \\
ACS/WFC & F850LP & 2.0 & 4045 & 26.0 & 26.7 \\
WFC3/IR & F105W & 1.0 & 2645 & 26.6 & 27.3 \\
WFC3/IR & F110W & 1.0 & 2415 & 27.0 & 27.8 \\
WFC3/IR & F125W & 1.0 & 2425 & 26.5 & 27.2 \\
WFC3/IR & F140W & 1.0 & 2342 & 26.7 & 27.4 \\
WFC3/IR & F160W & 2.0 & 4920 & 26.7 & 27.5 \\
\hline
\end{tabular}

havelarge Einstein radii. All of the CLASH clusters have Chandra observations, and 15 were also observed with XMM. 18 of the clusters show strong lensing arcs [1]. 

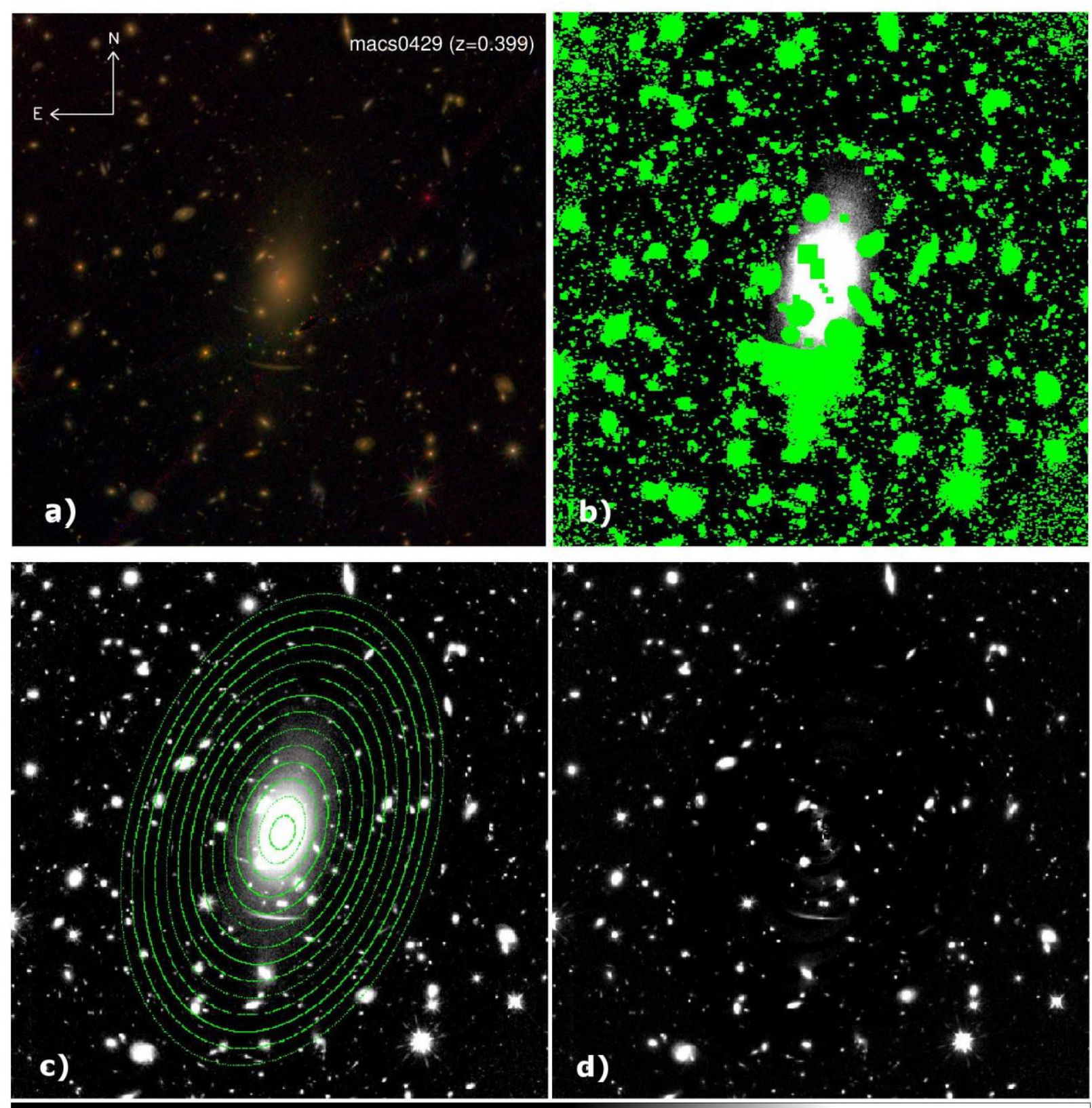

23

44

66

109

131

195

Fig. 1 An example of modeling and subtracting foreground dominated signal (MACS 0429.6-0253). a) Pseudo-color cut-out image made with observed $B, V, z$ images. b) H-band cut-out image overlapped with the 'MASK' image. c) H-band cut-out image overlapped with approximately elliptical isophotes (green ellipses). d) H-band cut-out image after subtracting our model created by using Fourier analysis. Each frame has 1800×1800 pixels.

Table 1 presents the names of sample clusters, positions, redshifts, and HST observing cycle and orbits. HST observes these clusters with totally 16 filters by the Advanced Camera for Surveys (ACS, [3]) and the Wide Field Camera 3 (WFC3,[4]), respectively. With continuous sampling of the broad wavelength range from the NUV to NIR that is enabled with WFC3 and ACS, The CLASH team we can obtain very accurate photometric redshifts of galaxies in each cluster, which helps to distinguish foreground light from faint background sources. In Table 2, we list all channels, filters, exposure time, and the limiting magnitudes for CLASH imaging. The image co-addition and alignment onto a grid with $0.065 \mathrm{arcsec} / \mathrm{pixel}$ is performed with an automated pipeline based on MosaicDrizzle [5]. Object detection and photometry is then accomplished using Sextractor [6] in dual image mode. 


\section{Modeling and Subtraction of Bright Foreground Source}

In this section, we illustrate the power of our modeling and subtracting foreground dominated signals with an example, MACS 0429.6-0253 (MACS0429, thereafter). As described above, CLASH observations cover 16 filters. The background levels in different filters are different. First, we use previously developed methods $[7,8]$ to perform the background subtraction in each filter independently. To obtain accurate information of the sky background, we generate a $1800 \times 1800$ pixels background only image using SExtractor [6] by masking all the detected objects with counts above 1- $\sigma$ noise in a frame smoothed by a circular Gaussian with a standard deviation $\sigma=3$ pixel. A median filter with $201 \times 201$ pixels is then convolved with the unmasked pixels, after which second order Legendre polynomials are used to fit both rows and columns, respectively [7]. The fitted Legendre polynomials are then further smoothed using a circular Gaussian filter with $\sigma=9$ pixel to obtain our final sky background model. We then subtract this model from the frame to obtain the background-free image.

In order to model foreground dominated source accurately, we mask other faint signals as illustrated in Panel b of Figure 1. We perform the Fourier analysis on each isophote of central dominated source. An ellipse is drawn to approximately match each isophote as illustrated in Panel c of Figure 1. The intensity along the ellipse is expanded in Fourier series

$$
I(\theta)=I_{0}+\sum\left(A_{n} \cos n \theta+B_{n} \sin n \theta\right)
$$

where $I_{0}$ is the intensity averaged over the ellipse, and $A_{n}$ and $B_{n}$ are the higher order Fourier coefficients. This analysis starts from a first guess elliptical isophote defined by approximate values for the $\mathrm{X}$ and $\mathrm{Y}$ center coordinates, ellipticity and position angle. Using these values, the image is sampled along an elliptical path producing a one-dimensional intensity distribution as a function of position angle. The harmonic content of this distribution is analyzed by least-squares fitting. After modeling the isophote in each annulus, we subtract all fitted ellipses from the background-free image to obtain a residual image. As illustrated in the Panel $d$ of Figure 1, our method is successful to remove bright foreground sources in the clusters, which significantly increases the effective depth of the images, and efficiently uncovers distant background signals in the centers of clusters.

\section{Summary}

The CLASH program offers an unprecedented opportunity to search for supernova at high redshifts and detect distant signals in the early universe. However, bright foreground light (mainly member galaxies and ICL) in the clusters prevents the detection and study of faint background sources. In this paper, we report a method of modeling and subtracting bright foreground sources. We perform the Fourier analysis on each isophote of foreground dominated galaxy in the clusters. After subtracting our modeled ellipses, faint sources behind foreground dominated galaxy are uncovered clearly. It crucially opens up the most magnified regions of the clusters.

\section{Acknowledgement}

This project was supported by the NSF grants of China (11103013, 11573017) and the Program for Liaoning Excellent Talents in University (LNET). The CLASH Multi-Cycle Treasury Program (GO-12065) is based on observations made with the NASA/ESA Hubble Space Telescope. The Space Telescope Science Institute is operated by the Association of Universities for Research in Astronomy, Inc. under NASA contract NAS 5-26555. ACS was developed under NASA contract NAS 5-32864. 


\section{References}

[1]M. Postman, D. Coe, N. Benítez, L. Bradley, T. Broadhurst, et al., The Astrophysical Journal Supplement, Volume 199, Issue 2, p.25-47 (2012).

[2] C. Burke, M. Hilton and C. Collins, Monthly Notices of the Royal Astronomical Society, Volume 449, Issue 3, p.2353-2367 (2015)

[3] H. C. Ford, M. Clampin, G. Hartig, G D. Illingworth, M. Sirianni, A. R. Martel, et al., Future EUV/UV and Visible Space Astrophysics Missions and Instrumentation. Edited by J. Chris Blades, Oswald H. W. Siegmund. Proceedings of the SPIE, Volume 4854, p. 81-94 (2003).

[4] R. A. Kimble, J. W. MacKenty, R. W. O'Connell and J. A. Townsend, Space Telescopes and Instrumentation 2008: Optical, Infrared, and Millimeter. Proceedings of the SPIE, Volume 7010, article id. 70101E, 12 pp. (2008).

[5] A. M. Koekemoer, A. S. Fruchter, R. N. Hook and W. Hack, The 2002 HST Calibration Workshop : Hubble after the Installation of the ACS and the NICMOS Cooling System, 337 (2003)

[6] E. Bertin and S. Arnouts, Astronomy and Astrophysics Supplement, v.117, p.393-404 (1996)

[7] F. S. Liu, X. Y. Xia, S. Mao, H. Wu and Z. G. Deng, Monthly Notices of the Royal Astronomical Society, Volume 385, Issue 1, p. 23-39 (2008)

[8] L. L. Qin, F. S. Liu, Y. Tu, G. Y. Yu and H. F. Yu, Image and Signal Processing, 2010 3rd International Congress, p.2458-2460 (2010) 